\title{
EXTENDED LAGUERRE INEQUALITIES AND A CRITERION FOR REAL ZEROS
}

\author{
DAVID A. CARDON
}

\begin{abstract}
Let $f(z)=e^{-b z^{2}} f_{1}(z)$ where $b \geq 0$ and $f_{1}(z)$ is a real entire function of genus 0 or 1 . We give a necessary and sufficient condition in terms of a sequence of inequalities for all of the zeros of $f(z)$ to be real. These inequalities are an extension of the classical Laguerre inequalities.
\end{abstract}

\section{INTRODUCTION}

The Laguerre-Pólya class, denoted $\mathcal{L P}$, is the collection of real entire functions obtained as uniform limits on compact sets of polynomials with real coefficients having only real zeros. It is known that a function $f$ is in $\mathcal{L P}$ if and only if it can be represented as

$$
f(z)=e^{-b z^{2}} f_{1}(z)
$$

where $b \geq 0$ and where $f_{1}(z)$ is a real entire function of genus 0 or 1 having only real zeros. The basic theory of $\mathcal{L P}$ can be found in [6, Ch. 8] and [8, Ch. 5.4].

In this paper, we extend a theorem of Csordas, Patrick, and Varga on a necessary and sufficient condition for certain real entire functions to belong to the LaguerrePólya class. They proved the following:

Theorem 1.1. Let

$$
f(z)=e^{-b z^{2}} f_{1}(z), \quad(b \geq 0, f(z) \not \equiv 0),
$$

where $f_{1}(z)$ is a real entire function of genus 0 or 1 . Set

$$
L_{n}[f](x)=\sum_{k=0}^{2 n} \frac{(-1)^{k+n}}{(2 n) !}\left(\begin{array}{c}
2 n \\
k
\end{array}\right) f^{(k)}(x) f^{(2 n-k)}(x)
$$

for $x \in \mathbb{R}$ and $n \geq 0$. Then $f(z) \in \mathcal{L P}$ if and only if

$$
L_{n}[f](x) \geq 0
$$

for all $x \in \mathbb{R}$ and all $n \geq 0$.

The forward direction is due to Patrick [7, Thm. 1]. The reverse direction was proved by Csordas and Varga [4, Thm. 2.9]. Theorem 1.1 is significant because it gives a nontrivial sequence of inequality conditions that hold for functions in the Laguerre-Pólya class. The case $n=1$ reduces to the classical Laguerre inequality which says that if $f(z) \in \mathcal{L P}$, then

$$
\left[f^{\prime}(x)\right]^{2}-f(x) f^{\prime \prime}(x) \geq 0
$$

Key words and phrases. Laguerre-Pólya class, real zeros, Laguerre inequalities. 
for $x \in \mathbb{R}$. Consequently, inequalities like those in Theorem 1.1 are sometimes called Laguerre-type inequalities. Csordas and Escassut discuss the inequalities $L_{n}[f](x) \geq 0$ and related Laguerre-type inequalities in [3]. Other results on similar inequalities of Turán and Laguerre types can be found in [1] and [2].

\section{An EXtension of Laguerre-type inequalities}

In this section, we extend Theorem 1.1 and give new necessary and sufficient inequality conditions for a function to belong to the Laguerre-Pólya class.

First we generalize the operator $L_{n}$ defined in Theorem 1.1. Let

$$
g(z)=\sum_{\ell=0}^{M} c_{\ell} z^{\ell}=\prod_{j=1}^{M}\left(z+\alpha_{j}\right)
$$

be a polynomial with complex roots. Define $\Phi(z, t)$ as the product

$$
\Phi(z, t)=\prod_{j=1}^{M} f\left(z+\alpha_{j} t\right)
$$

The coefficients of the Maclaurin series of $\Phi(z, t)$ with respect to $t$ are functions of $z$, and we write:

$$
\Phi(z, t)=\sum_{k=0}^{\infty} A_{k}(z) t^{k}
$$

where

$$
A_{k}(z)=\frac{1}{k !}\left[\frac{d^{k}}{d t^{k}} \Phi(z, t)\right]_{t=0}=\frac{1}{k !}\left[\frac{d^{k}}{d t^{k}} \prod_{j=1}^{M} f\left(z+\alpha_{j} t\right)\right]_{t=0} .
$$

Since $f(z)$ is entire, each $A_{k}(z)$ is entire. Another expression for $A_{k}(z)$ is given in (3.1). The choice $g(z)=1+z^{2}=(z-i)(z+i)$ produces $A_{2 k+1}(z)=0$ and $A_{2 k}(z)=L_{k}[f](z)$ as in (1.2) of Theorem 1.1. Thus, we may regard the sequence of functions $A_{k}(z)$ as a generalization of the sequence $L_{k}[f](z)$. We note that the zeros of $A_{k}(z)$ were studied by Dilcher and Stolarsky in [5]. In $\S 3$, we give several examples of $A_{k}(z)$ for interesting choices of $g(z)$.

Theorem 2.1. Let $f(z)=e^{-b z^{2}} f_{1}(z)$, where $f_{1}(z) \not \equiv 0$ is a real entire function of genus 0 or 1 and $b \geq 0$. Assume $g(z)$ in (2.1) is an even polynomial with nonnegative real coefficients having at least one non-real root. Then $f \in \mathcal{L P}$ if and only if

$$
A_{k}(x) \geq 0
$$

for all $x \in \mathbb{R}$ and all $k \geq 0$.

Corollary 2.2. The choice $g(z)=1+z^{2}$ in Theorem 2.1 gives $f(z) \in \mathcal{L P}$ if and only if $L_{k}[f](x) \geq 0$ for all $x \in \mathbb{R}$ and all $k \geq 0$, as stated in Theorem 1.1.

Proof of Theorem 2.1. Since $g(z)$ is an even polynomial, it follows that $\alpha_{j}$ is a root if and only if $-\alpha_{j}$ is a root with the same multiplicity. So,

$$
\Phi(z, t)=\prod_{j=1}^{M} f\left(z+\alpha_{j} t\right)=\prod_{j=1}^{M} f\left(z-\alpha_{j} t\right)=\Phi(z,-t) .
$$


Hence, $A_{k}(z) \equiv 0$ for all odd $k$ and we may write

$$
\Phi(z, t)=\sum_{k=0}^{\infty} A_{2 k}(z) t^{2 k} .
$$

Now assume $A_{2 k}(x) \geq 0$ for all $x \in \mathbb{R}$ and all $k \geq 0$. Let $f(z)=e^{-b z^{2}} f_{1}(z)$ where $b \geq 0$ and $f_{1}(z)$ is a real entire function of genus 0 or 1 , and assume $f(z)$ is not identically zero. Suppose, by way of contradiction, that $f(z)$ has a non-real root, say $z_{0}$. Let $\alpha_{s}$ be any fixed non-real root of $g(z)$ and write

$$
z_{0}=x_{0}+\alpha_{s} t_{0},
$$

where both $x_{0}$ and $t_{0}$ are real. Then $f\left(z_{0}\right)=f\left(x_{0}+\alpha_{s} t_{0}\right)=0$, and

$$
0=\Phi\left(x_{0}, t_{0}\right)=\prod_{j=1}^{M} f\left(x_{0}+\alpha_{j} t_{0}\right)=\sum_{k=0}^{\infty} A_{2 k}\left(x_{0}\right) t_{0}^{2 k} .
$$

Assume $t \neq 0$. Then the nonnegativity of $A_{2 k}\left(x_{0}\right)$ implies $A_{2 k}\left(x_{0}\right)=0$ for all $k$. This in turn implies $\Phi\left(x_{0}, t\right)$ is identically zero for all complex $t$. But that is false since $f(z)$ is a nonzero entire function. Therefore, $t_{0}=0$. Then $z_{0}=x_{0}+\alpha_{s} t_{0}=x_{0}$ is also real, contradicting the choice of $z_{0}$. Thus, all the roots of $f(z)$ are real and $f(z) \in \mathcal{L P}$.

Conversely, assuming $f(z) \in \mathcal{L P}$, we will show that $A_{2 k}(x) \geq 0$ for all $x \in \mathbb{R}$ and all $k \geq 0$. We will show this when $f(z)$ is a polynomial and the result for arbitrary $f(z) \in \mathcal{L P}$ will follow by taking limits. Let

$$
f(z)=\prod_{i=1}^{n}\left(z+r_{i}\right)
$$

where $r_{1}, \ldots, r_{n}$ are real. Calculating $\Phi(z, t)$ gives

$$
\begin{aligned}
\Phi(z, t) & =\prod_{j=1}^{M} f\left(z+\alpha_{j} t\right)=\prod_{j=1}^{M} \prod_{i=1}^{n}\left(z+\alpha_{j} t+r_{i}\right) \\
& =\prod_{i=1}^{n} \prod_{j=1}^{M}\left(\left(z+r_{i}\right)+\alpha_{j} t\right)=\prod_{i=1}^{n} \sum_{\ell=0}^{M} c_{\ell}\left(z+r_{i}\right)^{\ell} t^{M-\ell},
\end{aligned}
$$

where $g(z)=\prod_{j=1}^{M}\left(z+\alpha_{j}\right)=\sum_{\ell=0}^{M} c_{\ell} z^{\ell}$. Since $g(z)$ is an even polynomial, $c_{\ell}=0$ for odd $\ell$ and

$$
\Phi(z, t)=\prod_{i=1}^{n} \sum_{\ell=0}^{M / 2} c_{2 \ell}\left(z+r_{i}\right)^{2 \ell} t^{M-2 \ell}=\sum_{k=0}^{n M / 2} A_{2 k}(z) t^{2 k} .
$$

From (2.7), $A_{2 k}(z)$ is the sum of products of terms of the form $c_{2 \ell}\left(z+r_{i}\right)^{2 \ell}$. Because $c_{2 \ell} \geq 0$ and $\left(z+r_{i}\right)^{2 \ell}$ is a square, it follows that $A_{2 k}(x) \geq 0$ for real $x$.

Now let $f(z) \in \mathcal{L P}$ be an arbitrary function that is not a polynomial. Then there exist polynomials $f_{n}(z) \in \mathcal{L P}$ such that

$$
\lim _{n \rightarrow \infty} f_{n}(z)=f(z)
$$

uniformly on compact sets. The derivatives also satisfy

$$
\lim _{n \rightarrow \infty} f_{n}^{(k)}(z)=f^{(k)}(z)
$$


uniformly on compact sets. If we write

$$
\Phi_{n}(z, t)=\prod_{j=1}^{M} f_{n}\left(z+\alpha_{j} t\right)=\sum_{k=0}^{\infty} A_{n, 2 k}(z) t^{2 k},
$$

we see from (2.4) that

$$
\lim _{n \rightarrow \infty} A_{n, 2 k}(z)=A_{2 k}(z)
$$

uniformly on compact sets. Since $A_{n, 2 k}(x) \geq 0$ for real $x$, the limit also satisfies this inequality. Thus, for arbitrary $f(z) \in \mathcal{L P}, A_{2 k}(x) \geq 0$ for $x \in \mathbb{R}$ and $k \geq 0$, completing the proof of the theorem.

\section{Discussion And Examples}

The function $A_{k}(z)$ in (2.4) is described in terms of the $k$ th derivative of a product of entire functions. Either by using the generalized product rule for derivatives or by expanding each $f\left(z+\alpha_{j} t\right)$ as a series and multiplying series, one obtains the following formula for $A_{k}(z)$ :

$$
A_{k}(z)=\sum_{\lambda \vdash k} \frac{m_{\lambda}\left(\alpha_{1}, \ldots, \alpha_{M}\right)}{\lambda_{1} ! \cdots \lambda_{r} !} f(z)^{M-r} \prod_{j=1}^{r} f^{\left(\lambda_{j}\right)}(z),
$$

where $\lambda \vdash k$ means that the sum is over all unordered partitions $\lambda$ of $k$,

$$
\lambda=\left(\lambda_{1}, \ldots, \lambda_{r}\right) \quad k=\lambda_{1}+\cdots+\lambda_{r},
$$

where $r$ is the length of the partition $\lambda$, and where $m_{\lambda}\left(\alpha_{1}, \ldots, \alpha_{M}\right)$ is the monomial symmetric function of $M$ variables for the partition $\lambda$ evaluated at the roots $\alpha_{1}, \ldots, \alpha_{M}$.

The coefficients $c_{\ell}$ of $g(z)=\sum_{\ell=0}^{M} c_{\ell} z^{\ell}=\prod_{j=1}^{M}\left(z+\alpha_{j}\right)$ are elementary symmetric function of $\alpha_{1}, \ldots, \alpha_{M}$. The monomial symmetric functions $m_{\lambda}\left(\alpha_{1}, \ldots, \alpha_{M}\right)$ appearing in (3.1) can therefore be calculated in terms of $c_{0}, \ldots, c_{M}$ without direct reference to $\alpha_{1}, \ldots, \alpha_{M}$. We see that if $c_{0}, \ldots, c_{M}$ are real, then $m_{\lambda}\left(\alpha_{1}, \ldots, \alpha_{M}\right)$ is also real. However, in general $m_{\lambda}\left(\alpha_{1}, \ldots, \alpha_{M}\right)$ is not necessarily positive even if all the $c_{\ell}$ are positive. So, in the setting of Theorem 2.1, the type of summation appearing in (3.1) will typically involve both addition and subtraction, and the nonnegativity of $A_{k}(x)$ for real $x$ is not directly obvious from this representation.

Example 1. Let $g(z)=4+z^{4}$ and let $f(z) \in \mathcal{L P}$. Then $\Phi(z, t)$ is

$$
f(z+(1+i) t) f(z+(1-i) t) f(z+(-1+i) t) f(z+(-1-i) t)=\sum_{k=0}^{\infty} A_{2 k}(z) t^{2 k}
$$

A small calculation shows that

$$
\begin{aligned}
\frac{3}{2} A_{4}(x)=-f(z)^{3} f^{(4)}(z)+3 f(z)^{2} f^{\prime \prime} & (z)^{2}+6 f^{\prime}(z)^{4} \\
& +4 f(z)^{2} f^{(3)}(z) f^{\prime}(z)-12 f(z) f^{\prime}(z)^{2} f^{\prime \prime}(z) .
\end{aligned}
$$

According to Theorem 2.1 this expression is nonnegative for all $x \in \mathbb{R}$.

Example 2. Let $f(z)=\prod_{i=1}^{n}\left(z+r_{i}\right)$ where $r_{1}, \ldots, r_{n} \in \mathbb{R}$ and let

$$
g(z)=z^{2 m}+1=\prod_{j=1}^{2 m}\left(z+\omega^{2 j-1}\right)
$$


where $\omega=\exp (2 \pi i / 4 m)$ and $m \in \mathbb{N}$. Calculating as in (2.6) gives

$$
\begin{aligned}
\Phi(z, t) & =\prod_{i=1}^{n}\left(\left(z+r_{i}\right)^{2 m}+t^{2 m}\right) \\
& =f(z)^{2 m} \prod_{i=1}^{n}\left(1+\frac{t^{2 m}}{\left(z+r_{i}\right)^{2 m}}\right) \\
& =f(z)^{2 m} \sum_{k=0}^{n} e_{k}\left(\frac{1}{\left(z+r_{1}\right)^{2 m}}, \ldots, \frac{1}{\left(z+r_{n}\right)^{2 m}}\right)\left(t^{2 m}\right)^{k},
\end{aligned}
$$

where $e_{k}$ is the $k$ th elementary symmetric function of $n$ variables evaluated at $\left(z+r_{1}\right)^{-2 m}, \ldots,\left(z+r_{n}\right)^{-2 m}$. Thus, if $x \in \mathbb{R}$,

$$
A_{2 m k}(x)=f(x)^{2 m} e_{k}\left(\frac{1}{\left(x+r_{1}\right)^{2 m}}, \ldots, \frac{1}{\left(x+r_{n}\right)^{2 m}}\right)
$$

is expressed as a sum of squares of real numbers and is therefore nonnegative. Dilcher and Stolarsky studied the zeros of $A_{2 m k}(x)$. (See Prop. 2.3 and $\S 3$ of [5]).

Example 3. This example illustrates how certain modifications to Theorem 2.1 are possible. Let $f(z)$ be a polynomial with negative roots. Then $f(z)=\prod_{i=1}^{n}\left(z+r_{i}\right)$ where each $r_{i}>0$. Let

$$
g(z)=1+z+z^{2}=\left(z+e^{\pi i / 3}\right)\left(z+e^{-\pi i / 3}\right) .
$$

Although $g(z)$ is not even as in the hypothesis of the theorem, its coefficients are nonnegative. Then

$$
\begin{aligned}
\Phi(z, t)= & f\left(z+t e^{\pi i / 3}\right) f\left(z+t e^{-\pi i / 3}\right) \\
= & \underbrace{f(z)^{2}}_{A_{0}(z)}+\underbrace{f(z) f^{\prime}(z)}_{A_{1}(z)} t+\underbrace{\frac{1}{2 !}\left(2 f^{\prime}(z)^{2}-f(z) f^{\prime \prime}(z)\right)}_{A_{2}(z)} t^{2} \\
& +\underbrace{\frac{1}{3 !}\left(3 f^{\prime}(z) f^{\prime \prime}(z)-2 f(z) f^{\prime \prime \prime}(z)\right)}_{A_{3}(z)} t^{3} \\
& +\underbrace{\frac{1}{4 !}\left(6 f^{\prime \prime}(z)^{2}-4 f^{\prime}(z) f^{(3)}(z)-f(z) f^{(4)}(z)\right)}_{A_{4}(z)} t^{4}+\cdots
\end{aligned}
$$

On the other hand, calculating as in (2.6) gives

$$
\begin{aligned}
\Phi(z, t) & =\prod_{i=1}^{n}\left(\left(z+r_{i}\right)^{2}+\left(z+r_{i}\right) t+t^{2}\right) \\
& =f(z)^{2} \prod_{i=1}^{n}\left(1+\frac{t}{z+r_{i}}+\frac{t^{2}}{\left(z+r_{i}\right)^{2}}\right) \\
& =f(z)^{2} \sum_{k=0}^{2 n}\left(\sum_{\substack{\lambda \vdash k \\
\lambda_{j} \leq 2}} m_{\lambda}\left(\frac{1}{z+r_{1}}, \ldots, \frac{1}{z+r_{n}}\right)\right) t^{k},
\end{aligned}
$$

where the inner sum is over all unordered partitions $\lambda$ of $k$ whose parts satisfy $\lambda_{j} \leq 2$ and where $m_{\lambda}$ is the monomial symmetric function in $n$ variables for the partition $\lambda$ evaluated at $\left(z+r_{1}\right)^{-1}, \ldots,\left(z+r_{n}\right)^{-1}$. From the last expression, we see that each

$$
A_{k}(x) \geq 0
$$


for all $x \geq 0$ and all $k \geq 0$.

\section{REFERENCES}

[1] David A. Cardon and Adam Rich, Turán inequalities and subtraction-free expressions, JIPAM. J. Inequal. Pure Appl. Math. 9 (2008), no. 4, Article 91, 11 pp. (electronic).

[2] Thomas Craven and George Csordas, Iterated Laguerre and Turán inequalities, JIPAM. J. Inequal. Pure Appl. Math. 3 (2002), no. 3, Article 39, 14 pp. (electronic).

[3] George Csordas and Alain Escassut, The Laguerre inequality and the distribution of zeros of entire functions, Ann. Math. Blaise Pascal 12 (2005), no. 2, 331-345.

[4] George Csordas and Richard S. Varga, Necessary and sufficient conditions and the Riemann hypothesis, Adv. in Appl. Math. 11 (1990), no. 3, 328-357.

[5] Karl Dilcher and Kenneth B. Stolarsky, On a class of nonlinear differential operators acting on polynomials, J. Math. Anal. Appl. 170 (1992), no. 2, 382-400.

[6] Boris Ja. Levin, Distribution of zeros of entire functions, revised ed., Translations of Mathematical Monographs, vol. 5, American Mathematical Society, Providence, R.I., 1980.

[7] Merrell L. Patrick, Extensions of inequalities of the Laguerre and Turán type, Pacific J. Math. 44 (1973), 675-682.

[8] Qazi I. Rahman and Gerhard Schmeisser, Analytic theory of polynomials, London Mathematical Society Monographs. New Series, vol. 26, The Clarendon Press Oxford University Press, Oxford, 2002.

Department of Mathematics, Brigham Young University, Provo, Utah 84602, USA, E-MAIL: CARDON@MATH.BYU.EDU, HTTP://WWw.MATH.BYU.EDU/CARDON 